\title{
Denkanstöße für eine integrierte Krisenvorsorge
}

Vor dem Hintergrund der COVID-19-Pandemie stellt sich auch für Unternehmen die Frage, wie sie sich im Sinne einer Krisenvorsorge auf unerwartete, aber dennoch mögliche Ereignisse vorbereiten können. Ein Blick auf unterschiedliche Krisen-Management-Ansätze kann helfen, neue Weichen zu stellen.

Ulrich Krystek 
Es muss wohl als sicher gelten, dass die COVID-19-Pandemie die folgenschwerste Krise seit Ende des Zweiten Weltkrieges für eine Vielzahl von Unternehmen darstellt. Unter Krise ist ein überlebenskritischer Prozess mit ambivalentem Ausgang zu verstehen. In dessen Verlauf verringert sich seine Beeinflussbarkeit, während sich die von ihm ausgehenden Bedrohungen verstärken. Der Krisenprozess ist in vier Phasen unterteilbar: die potenzielle Krise, die latente Krise, die akut/beherrschbare Krise und schließlich die akut/nicht beherrschbare Krise.

Die Phaseneinteilung des generellen Krisenprozesses erlaubt eine Zuordnung von Aktionen zur Vermeidung oder Bewältigung von Unternehmenskrisen (vergleiche Krystek/Moldenhauer 2007, S. 34 ff.). Unter dem Aspekt der Krisenvorsorge kommt den beiden ersten Phasen eine entscheidende Bedeutung zu. In der Phase der potenziellen, lediglich möglichen und in ihren Wirkungen noch nicht vorhandenen Krise können durch gedankliche Vorwegnahme unerwarteter, jedoch möglicher Ereignisse geeignete Vorsorgestrategien und -maßnahmen entwickelt werden. In der Phase der latenten Krise wird mithilfe von Frühwarn-/Früherkennungssystemen eine rechtzeitige Wahrnehmung der verdeckt bereits vorhandenen Krise ermöglicht (vergleiche Krystek/ Moldenhauer 2007, S. 97 ff.). In der dritten und vierten Phase geht es um Krisenbewältigung. Befindet sich ein Unternehmen in der Phase der akut/beherrschbaren Krise, sind die von ihr ausgehenden Wirkungen für das Unternehmen direkt spürbar. Dominante Ziele - wie beispielsweise Ergebnis und/ oder Liquidität - sind akut gefährdet, doch ist eine konstruktive Bewältigung der Krise durch Restrukturierung oder Sanierung noch möglich, sogar noch in der Insolvenz. Gelingt eine Rettung des Unternehmens nicht, so tritt der Krisenprozess in seine letzte Phase, die akut/nicht beherrschbare Krise. Diese endet mit dem Untergang des Unternehmens in seiner bisherigen Form.

Ein umfassendes Krisen-Management sollte alle vier Phasen des Krisenprozesses abdecken. Es muss damit neben einem reaktiven Ad-hoc-KrisenManagement auch ein auf Krisenvorsorge und -früherkennung ausgerichtetes, aktives Krisen-Management beinhalten (vergleiche Abbildung 1).

\section{Krisenvorsorge im Rahmen des Krisen-Managements}

Häufig wird die derzeitige Pandemie als ein „Black Swan“ und damit als ein Ereignis bezeichnet, das unvorhersehbar auftritt und auf das man sich daher nicht vorbereiten kann. Zwar ist sie tatsächlich ein sehr seltenes, unerwartetes (nicht als wahrscheinlich angenommenes) Ereignis mit extrem destruktiven, überlebenskritischen Wirkungen. Allerdings war die Möglichkeit einer Pandemie an sich als potenzielle Krise vorhersehbar (vergleiche Wucker 2020; GMPB 2019). So hat beispielsweise Bill Gates bereits 2015 in einem Interview zum Thema „The next outbreak? We're not ready" vor dem Ausbruch einer solchen Pandemie gewarnt (vergleiche Harford 2020, S. 3). Die derzeitige Corona-Pandemie war durch die von ihr ausgehenden schwachen Signale (Weak Signals) einer Früherkennung zugänglich. Schließlich gilt sie, so ist zu hoffen und zu erwarten, als eine akut/beherrschbare Krise. Sie ist jedoch kein „Black Swan“ im Sinne von Nassim N. Taleb (2007), der diesen Begriff populär gemacht hat. Vielmehr

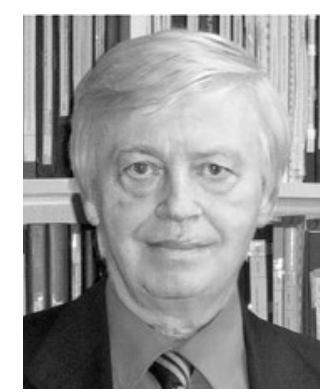

Prof. Dr. Ulrich Krystek

war bis 2018 Leiter des Lehrgebietes Strategisches Controlling an der Technischen Universität Berlin, Fakultät VII, Wirtschaft und Management. E-Mail:u.krystek@web.de 


\section{Krisenvorsorge gehört zu den elementaren Aufgaben der Führung, steht jedoch nicht immer weit oben auf deren Agenda.}

deutet dieses aktuelle Beispiel die Möglichkeiten eines umfassenden KrisenManagements und der Krisenvorsorge in seinem Rahmen an.

In einer von der WHU - Otto Beisheim School of Management in Zusammenarbeit mit dem Internationalen Controller Verein im März 2020 durchgeführten Blitzumfrage unter CFOs und Controllern wurde deutlich, dass nicht Ad-hoc-Reaktionen, sondern eine bestmögliche Vorbereitung ein gutes KrisenManagement ausmacht (vergleiche Reimer/Schäffer/Weber 2020). Zugleich betonte die Hälfte der Befragten allerdings auch den Chancen-Aspekt und damit die positive Sichtweise auf die gegenwärtige Krise (vergleiche Ludowig 2020, S. 21). Dennoch: Krisenvorsorge kann wohl noch immer als ein Themenbereich gesehen werden, der in der Praxis nicht sehr weit oben auf der Agenda von Controlling und Management steht. Ein damit verbundener Mehraufwand erklärt dies ebenso wie die Tatsache, dass sich solche Aufwendungen möglicherweise niemals ,,auszahlen“. Die geringe Eintrittswahrscheinlichkeit und Dringlichkeit hemmen zudem offenbar eine Realisierung der grundsätzlich durchaus als wichtig anerkannten Krisenvorsorge (vergleiche Harford 2020, S. 3 ff.).

Zwei scheinbar konkurrierende Ansätze der Krisenvorsorge sind zu beachten: zum einen Ansätze, die die Erhaltung beziehungsweise Wiederherstellung des bisherigen Zustandes von Unternehmen (Status quo) in akuten Krisen in den Fokus stellen, und zum anderen Ansätze, die eine robuste, widerstandsfähige Ausgestaltung von Unternehmen (funktionaler Zielzustand) bei potenziellen Krisen zum Ziel haben.

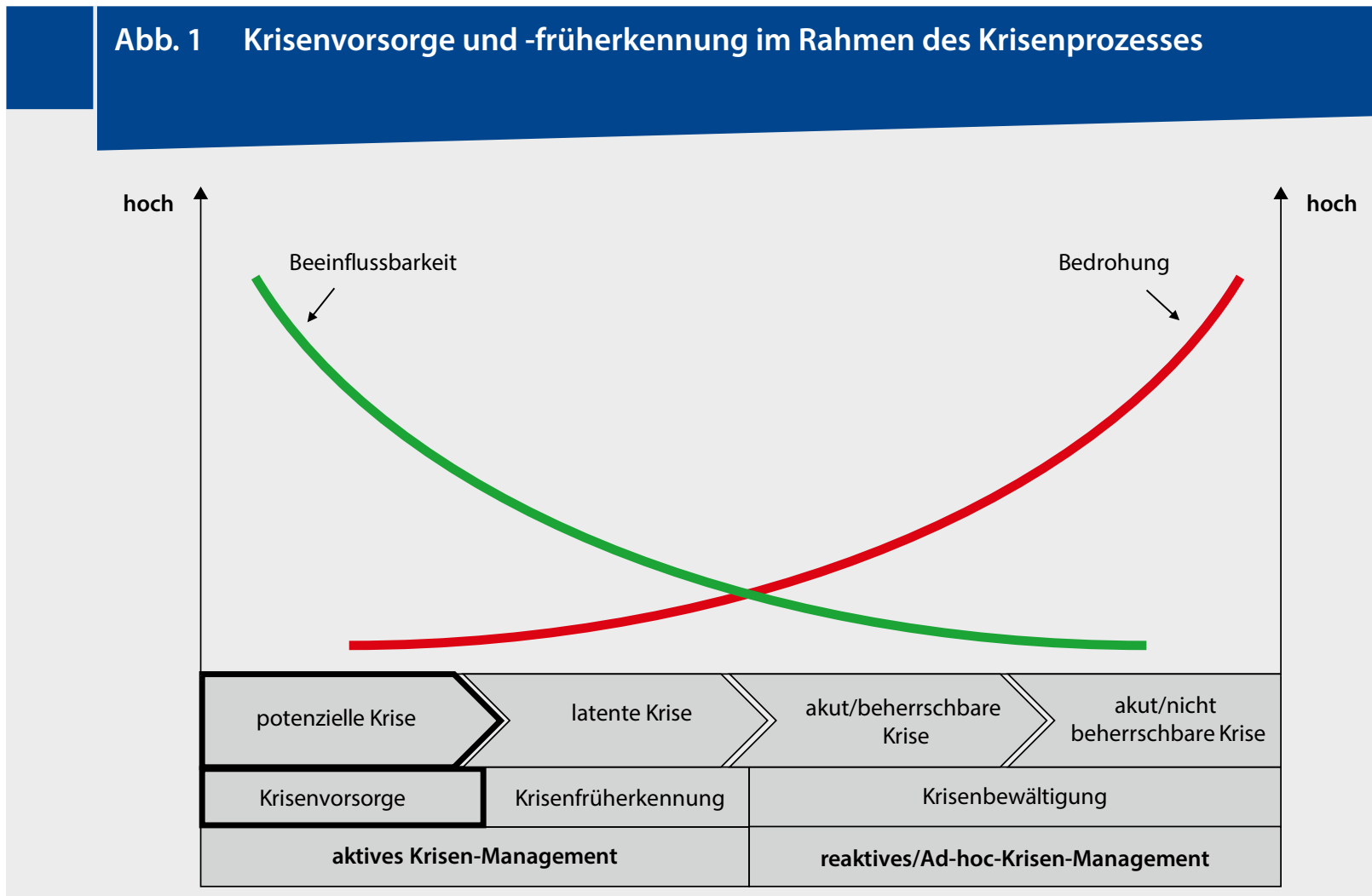

Quelle: eigene Darstellung 


\section{Den Status quo von Unternehmen sichern}

\section{Risiko-Management}

$\mathrm{Zu}$ den traditionellen Formen der Vorsorge (auch) gegenüber bestandsgefährdenden Risiken zählt zunächst das Risiko-Management gemäß $\$ 91$ Absatz 2 Aktiengesetz (KonTraG). Das Gesetz schreibt die Einführung eines Überwachungssystems zur Früherkennung von bestandsgefährdenden Entwicklungen vor. Im Fokus steht ein an Eintrittswahrscheinlichkeiten orientiertes Vorgehen, nicht eines, das auf unerwartete Ereignisse eingeht. Laut einer Umfrage von 2018 verfügten die befragten Unternehmen zwar mehrheitlich über ein Risiko-Management, 71 Prozent gaben jedoch an, damit hauptsächlich die rechtlichen Vorschriften zu erfüllen (vergleiche Ulrich/Barth/Lehmann 2018, S. 154 ff.). Lediglich zwölf Prozent der befragten Unternehmen verfügten tatsächlich über ein strategisches Früherkennungssystem.

\section{Contingency Planning}

Insbesondere in den Bereichen der inneren und äußeren Sicherheit sowie im Bereich des Katastrophenschutzes wird Contingency Planning (Alternativplanung/Eventualplanung/Schubladenplanung/„Plan B“) seit langer Zeit erfolgreich angewendet. Abbildung 2 zeigt den dazugehörigen Prozess mit den notwendigen informationellen Input-Faktoren. Gegenstände des Contingency Planning sind - im Gegensatz zur regulären Unternehmenspla-

\section{Zusammenfassung}

- Vorsorge und Achtsamkeit gegenüber unerwarteten, aber möglichen Krisen sind generell einem Ad-hoc-KrisenManagement überlegen. Dies zeigen gerade auch die aktuellen Ereignisse.

- Unterschiedliche Ansätze der Krisenvorsorge sind zwar bekannt, werden aber in der Praxis noch nicht hinreichend berücksichtigt und sind zu wenig miteinander vernetzt.

- Aus einer Analyse der bekannten Ansätze zur Krisenvorsorge lassen sich konkrete Denkanstöße für Controlling und Management für eine integrierte Krisenvorsorge ableiten.

\section{Abb. 2 Prozess Contingency Planning mit informationellen Input-Faktoren}

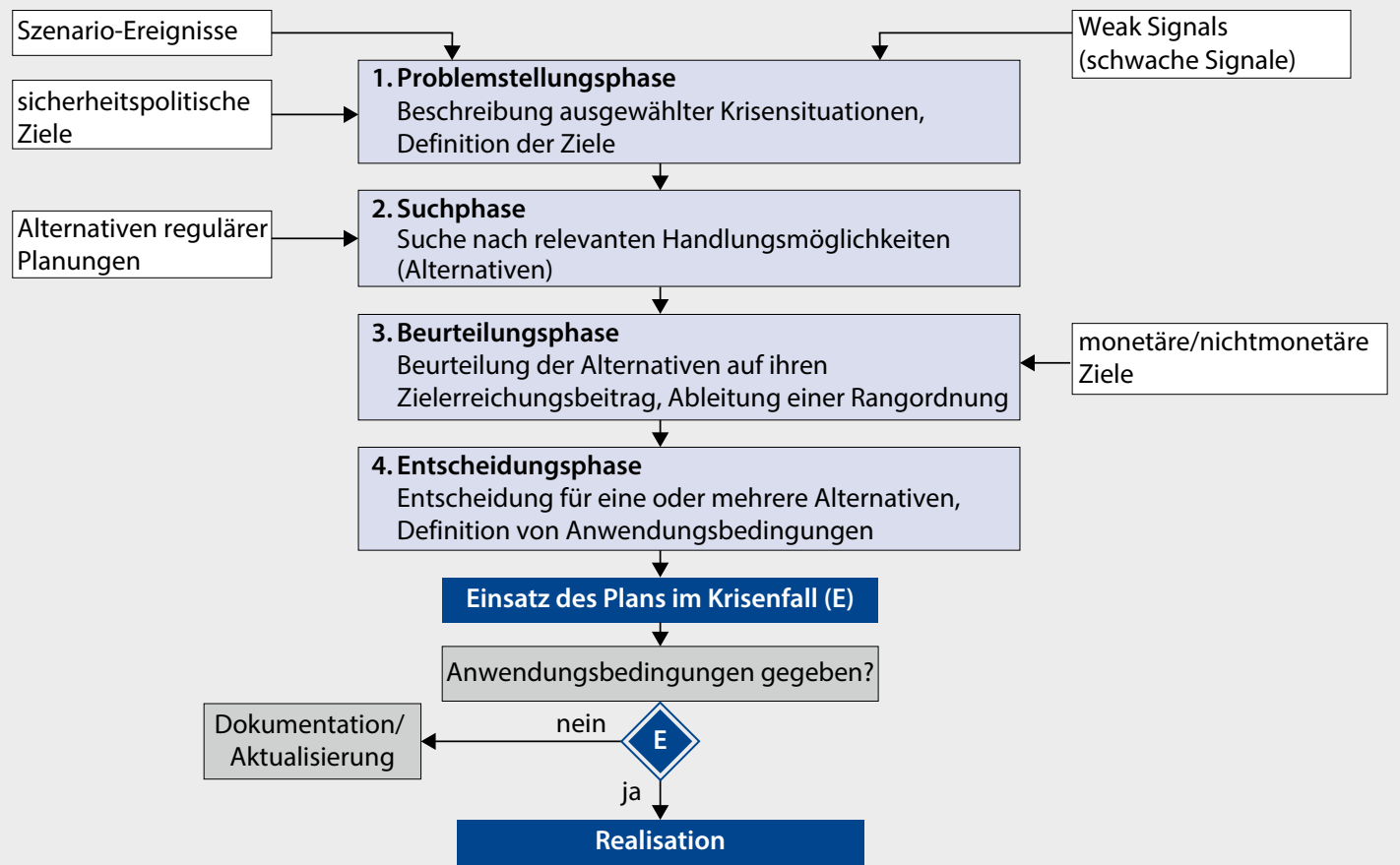

Quelle: eigene Darstellung 


\section{Gegenüber möglichen Krisen muss im Unternehmen ein Klima der Dringlichkeit erzeugt und wach gehalten werden.}

nung - unerwartete Ereignisse mit großem Krisenpotenzial. Dazu gehören im unternehmensexternen Bereich etwa die Insolvenz wichtiger Kunden/ Lieferanten, Export-/Importverbote und Strafzölle, Streiks/Bürgerkriege/ Terroranschläge, Pandemien sowie die Entwicklung von Konkurrenz-Technologien. Im unternehmensinternen Bereich zählen dazu beispielsweise der Ausfall wichtiger Führungskräfte, Werkspionage, Hackerangriffe, der Ausfall von IT-Systemen oder feindliche Übernahmen.

Solche möglichen Gegenstände können jeweils nur unternehmensindividuell ermittelt werden. Doch gerade aus dem Prozess des Durchdenkens unerwarteter Entwicklungsmöglichkeiten ergibt sich ein erheblicher Erkenntnisgewinn für das Unternehmen. Zu beachten ist allerdings, dass sich relevante Gegenstände des Contingency Planning im Zeitablauf verändern können. Weiterhin sind die ermittelten Gegenstände mit den Kernaussagen der regulären Unternehmensplanung hinsichtlich sicherheitspolitischer Ziele (zum Beispiel Risikobereitschaft) sowie übriger monetärer und nichtmonetärer Zielsetzungen (Ergebnis, Liquidität, Strategien) einschließlich der daraus abzuleitenden Maßnahmen abzugleichen. Schließlich besteht eine wechselseitige Informationsbeziehung zwischen beiden Planungen: Zum einen setzt der Wechsel zu einem „Plan B“ die Kenntnis der Inhalte bisher gültiger (regulärer) Planungen voraus. Zum anderen können aber auch Annahmen und Inhalte von Contingency Plans neue Impulse für reguläre Planungen geben.

\section{„Der durch Krisenvorsorge erreichte Handlungs- vorsprung kann entscheidend sein."}

Contingency Planning ermöglicht es Unternehmen, sich - unter anderem auf Basis von Weak Signals und einer darauf aufbauenden, strategischen Früherkennung sowie unter Anwendung der Szenario-Technik - auf unerwartete, überlebenskritische Ereignisse durch Pläne vorzubereiten. So können sie im Eintrittsfall eines Ereignisses Zeit gewinnen. Der dadurch erreichte und gegebenenfalls entscheidende Handlungsvorsprung rechtfertigt unter dem Aspekt der Krisenvorsorge den damit verbundenen zusätzlichen Aufwand (vergleiche Krystek/Moldenhauer 2007, S. 95).

\section{Business Continuity Management}

Die zentrale Aufgabe des Business Continuity Managements (BCM) besteht in der Sicherung und Fortführung der Geschäftstätigkeit von Unternehmen unter überlebenskritischen Bedingungen. Während in Deutschland unter der Bezeichnung „Betriebliches Kontinuitäts-Management“ erst in jüngerer Zeit und noch keinesfalls flächendeckend mit der Einführung dieses Rahmenkonzeptes begonnen wurde, hat sich das BCM in den angloamerikanischen Ländern fest etabliert und in den USA zur Gründung des Business Continuity Institutes geführt. Die mittlerweile durch die ISO-Norm 22301 
international vereinheitlichten Schritte des BCMs sind in Abbildung 3 dargestellt (vergleiche nachfolgend Krystek 2014, S. 377 ff.):

In Schritt 1 werden auf Basis einer Analyse der kritischen Geschäftsaktivitäten von Unternehmen unter Berücksichtigung sicherheitspolitischer Zielsetzungen zunächst grundlegende Kontinuitätsstrategien abgeleitet. Diese gewährleisten in Schritt 2 eine strategische Absicherung der gegenwärtigen Geschäftsaktivitäten des Unternehmens auch in Krisensituationen. Daraus sind in Schritt 3 konkrete Handlungsanweisungen in Form von spezifizierten BCM-Plänen zu entwickeln und zu implementieren. Wesentlich für den Erfolg des BCMs sind Schritt 4, der Aufbau sowie die Verankerung einer BCM-Kultur, sowie Schritt 5, die explizit geforderte Notwendigkeit einer wiederkehrenden Übung, Wartung und Revision der Pläne. Alle diese Schritte und Maßnahmen im Rahmen des BCM werden durch ein Programm-Management koordiniert.

\section{Unternehmen widerstandsfähig ausgestalten}

\section{Resilienz}

Das Thema Resilienz wird derzeit in der Psychologie und in den Sozialwissenschaften intensiv diskutiert. Daraus wurde der Ansatz des organisationalen Resilienz-Managements entwickelt. Ziel ist es, durch neu gestaltete Führungsprinzipien und Strategien unter akuten Krisenbedingungen nicht

\section{Bekannte Ansätze der Krisen- vorsorge sind unternehmens- individuell auszugestalten und auszubauen.}

\section{Abb. 3 Schritte des Business Continuity Managements (BCM)}

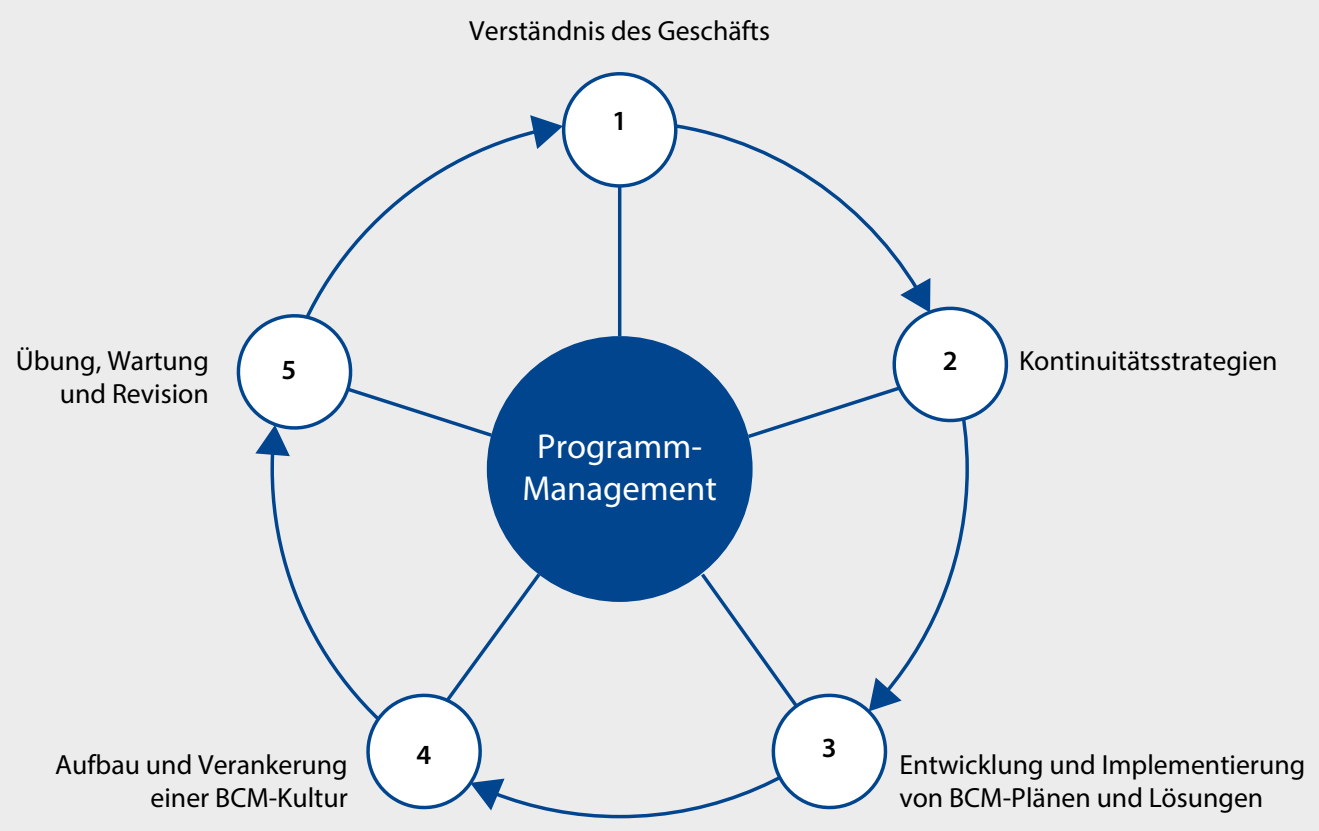


lediglich den bisherigen Normalzustand, sondern von vornherein einen funktionalen, gegenüber potenziellen Krisen widerstandsfähigen Zielzustand bei Unternehmen zu erreichen (vergleiche Meissner/Hunziker 2017, S. 14 f.). Die Kernelemente des Resilienz-Managements (vergleiche Abbildung 4) integrieren zugleich Grundprinzipien von Hochzuverlässigkeitsorganisationen (High Reliability Oganizations - HRO). Solche HROs, zu denen zum Beispiel Feuerwehr, Polizei oder Flugsicherungszentralen zählen, streben nach Flexibilität und Resilienz. Besondere Bedeutung kommt bei HROs einer sogenannten Strategie der Redundanz zu, mit der kritische Ressourcen als Puffer bewusst mehrfach vorgehalten werden sollen, redundante Kontrollen und Überwachungen stattfinden sowie auch redundante Entscheidungsfindungskanäle zur Verfügung stehen. Ebenso geht es um eine Achtsamkeit gegenüber (auch seltenen) Bedrohungen bereits durch Berücksichtigung der von ihnen zumeist ausgehenden Weak Signals. Als Instrumente empfehlen sich auch für diesen Ansatz neben einer strategischen Früherkennung die Szenario-Technik sowie das Contingency Planning (vergleiche Krystek 2014, S. 384 ff.).

\section{Antifragilität}

Der von Taleb (2012) eingeführte Ansatz der „Antifragilität“ kann nicht als ein Management-Konzept gelten. Er ist vielmehr eine sehr breit ausgelegte, spekulative und provokative „Anleitung für eine Welt, die wir nicht verste-

\section{Abb. 4 Kernelemente des Resilienz-Managements}

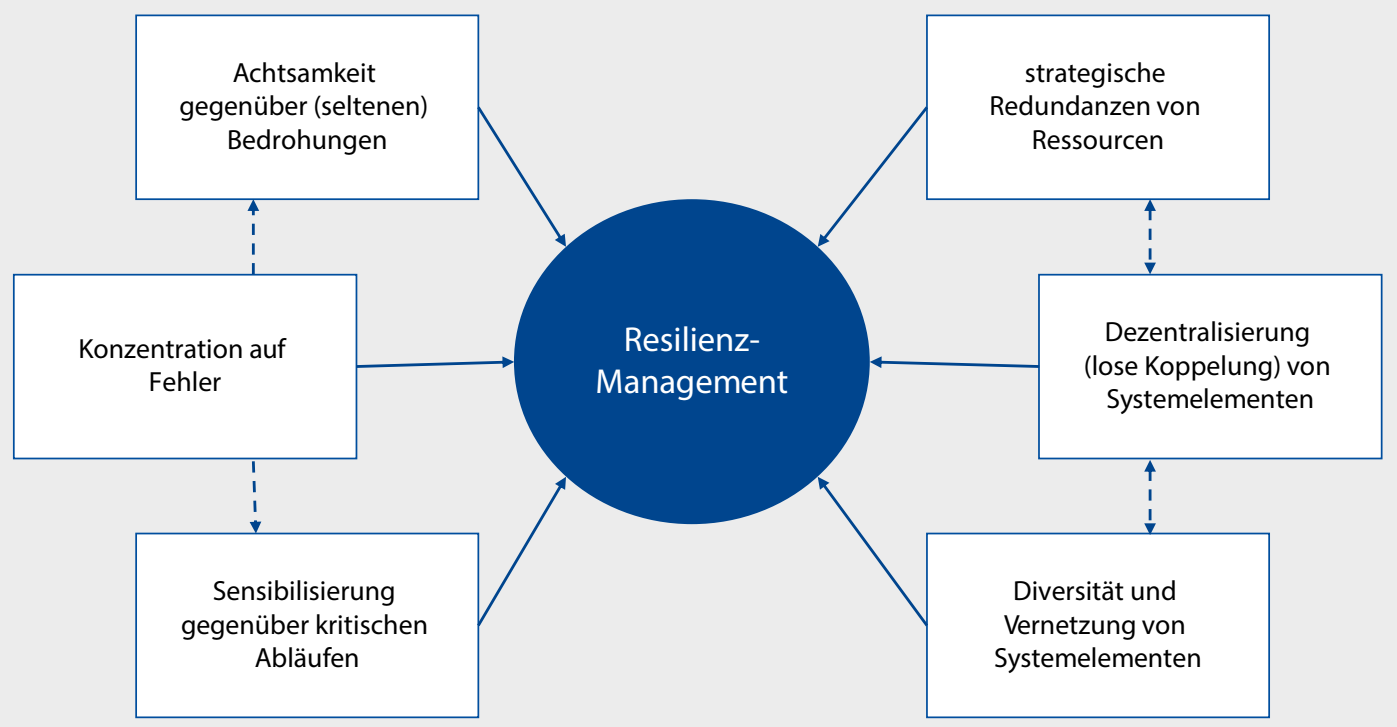

Führungsprinzipien

Strategien 
hen“, wie es im Untertitel der deutschen Ausgabe (2013) heißt. Allerdings enthält sein Werk durchaus auch interessante Ideen, die für Ansätze der Krisenvorsorge beachtlich erscheinen.

Antifragilität wird aus der Fragilität komplexer Systeme - und damit auch von Unternehmen - heraus verständlich, die zerbrechlich im Sinne einer überlebenskritischen Störanfälligkeit sind. Der durch die aktuellen Ereignisse bewirkte Zusammenbruch von internationalen Lieferketten kann dafür als Beispiel gelten. Neben den bekannten Elementen der Achtsamkeit, Redundanz und einer Abkehr von Größe durch Dezentralisierung (lose Koppelung) enthält dieser Ansatz durchaus innovative Denkanstöße, die mit den Begriffen Prognose- und Planungspessimismus sowie Optionalität umrissen sind (vergleiche ausführlich Krystek 2014, S. 390 ff.).

Antifragile Systeme sehen Trend-Extrapolationen, die speziell auf Hochrechnungen aus der Vergangenheit beruhen, äußerst kritisch (Prognosepessimismus). Diese verleiten in trügerischem Vertrauen auf die Vorhersagbarkeit nur einer Zukunftsentwicklung dazu, überlebenskritische Risiken einzugehen. Damit in Verbindung stehend wendet sich der Ansatz der Antifragilität auch gegen detaillierte und optimierte, langfristige Planungen. Sie engen nach Taleb den Handlungsspielraum von Unternehmen vor dem Hintergrund unvorhersehbarer und zufälliger Umfeldbedingungen in bedrohlicher Weise ein (Planungspessimismus). Anstelle einer Fixierung auf Planungen gilt es, neue Gelegenheiten als Optionen aufzugreifen (Optionalität). Sie sollten nach der von Taleb konstruierten Figur des „rationalen Flaneurs“ flexibel genutzt werden. Der „rationale Flaneur“ ist - im Gegensatz zu den sich ausschließlich an ihren Reiseführern orientierenden Touristen - nicht Gefangener eines kaum noch zu revidierenden Plans, sondern er kann sich bietende Gelegenheiten spontan ergreifen und von vorgegebenen Routen abweichen.

\section{Auf dem Weg zu einer integrierten Krisenvorsorge}

Ein umfassendes Krisen-Management sollte die aufgeführten Ansätze kennen und darauf aufbauend eine für das eigene Unternehmen passgenaue, integrierte Krisenvorsorge ableiten und ausgestalten. Die Integration der Ansätze könnte dabei in zwei Schritten erfolgen.

Zunächst gilt es, die auf Sicherung beziehungsweise Wiederherstellung des Status quo abstellenden Ansätze (Status-quo-Ansätze) stärker miteinander zu verzahnen. Grundpfeiler bleibt das traditionelle Risiko-Management, das jedoch nicht nur der Erfüllung gesetzlicher Vorgaben dienen, sondern als gelebtes Instrument der Krisenvorsorge weiterentwickelt werden sollte. Als Orientierungshilfe für eine Integration der übrigen Status-quo-Ansätze kann der Risiko-Management-Prozess dienen.

Für die Identifikation und Analyse von (überlebenskritischen) Risiken ist der verstärkte und systematische Einsatz von Szenarien und Frühwarn-/ Früherkennungssystemen zu empfehlen. Bei der Risikobewertung sind Erkenntnisse des Business Continuity Managements zu berücksichtigen, das unabhängig von Eintrittswahrscheinlichkeiten - den Fokus auf eine unter
Controlling verfügt über alle notwendigen Kompetenzen, um Krisenvorsorge systematisch zu betreiben und auszubauen. 
Krisenbedingungen gewährleistete Fortführung von kritischen Geschäftsprozessen legt. Von größter Bedeutung ist dabei der Einsatz von Contingency Plans im Rahmen der Risikosteuerung. Sie ermöglichen es, auf Basis von (Worst Case-)Szenarien Handlungsoptionen zu ermitteln und vorzuhalten, die im Bedarfsfall sehr schnell verfügbar sind (vergleiche Neuhaus 2006, S. 416). Sinnvoll sind zudem Prozesskontrollen. Sie gewährleisten, dass Informationen aus Frühwarn-/Erkennungssystemen während des gesamten Risiko-Management-Prozesses zur Verfügung stehen und das Unternehmen so frühzeitig auf kritische Abweichungen reagieren kann.

Die Integration der auf eine robuste beziehungsweise widerstandsfähige Unternehmensgestaltung ausgelegten Ansätze könnte in einem zweiten Schritt erfolgen. Hier geht es um eine eher langfristige und grundlegende Neugestaltung von Strategien und Führungsprinzipien, wie sie aus dem Resilienz-Management abzuleiten sind. Das allerdings braucht Zeit, da zu ihrer wirksamen Realisierung Elemente der bestehenden Unternehmenskultur angepasst werden müssen.

Das Prinzip der Achtsamkeit gegenüber unerwarteten, krisenträchtigen Ereignissen sollten Controller und Manager in ihr Leitbild aufnehmen und zum Bestandteil der Unternehmenskultur werden lassen. Dies allerdings hat ökonomische Konsequenzen. Achtsamkeit als Ziel steht - jedenfalls bei kurzfristiger Betrachtung - in einem erheblichen Spannungsverhältnis zu ökonomischen Zielen, wie etwa dem der Ergebnisoptimierung. Ein solches Spannungsverhältnis gilt es, im Hinblick auf eine nachhaltige Sicherung und erfolgreiche Weiterentwicklung von Unternehmen auszutarieren: gewiss schwierig, aber möglich und wohl auch notwendig.

Mit einer Veränderung der Planungskultur verbunden ist die Anerkennung und Berücksichtigung von Grenzen der Planung, die der Idee von Antifragilität entnommen werden kann. Anstelle von zu detaillierten und optimierten Planungen sollten robuste Programme entworfen werden, die auch unter unerwarteten, krisenhaften Bedingungen erfolgreich sind (vergleiche Neuhaus 2006, S. 418).

Auch wenn die robusten Ansätze der Krisenvorsorge langfristig eine bessere Absicherung gegenüber potenziellen Krisen versprechen, sie sind weit weniger konkret und ersetzen keinesfalls den Ausbau der Status-quo-Ansätze. Vielmehr ergänzen sie diese und führen in Kombination zu einem integrierten Konzept der Krisenvorsorge.

\section{Schlussbetrachtung}

Die COVID-19-Pandemie gibt Anlass, das Thema der Krisenvorsorge wieder stärker in den Blick zu nehmen. Die Ansätze dazu sind bekannt, haben aber in der Anwendung durch Unternehmen noch eher fragmentarischen Charakter. Vielleicht auch deshalb führen sie - mit Ausnahme des RisikoManagements - eher noch ein Schattendasein in der Unternehmenspraxis. Hier ist das Controlling gefordert. Zentrale Elemente der Krisenvorsorge sind bereits in seinem Aufgabenspektrum angelegt. In seiner Informationsversorgungs- und Rationalitätssicherungs-Funktion bietet sich das Control- 
ling für diese herausfordernde Aufgabe an. Zudem ist ihm der Umgang mit Risiken keinesfalls neu, und Controlling beinhaltet immer auch ein Controlling von Risiken.

Es gilt daher, die dargestellten Ansätze passgenau für das Unternehmen zu integrieren, um so - in der Mitverantwortung für das Unternehmen einen wichtigen Beitrag zur Krisenvorsorge zu leisten. Eine Controlling-Aufgabe, die zunehmend an Bedeutung gewinnen dürfte.

\section{Literatur}

GPMP - Global Preparedness Monitoring Board (2019): A World at Risk. Annual Report on Global Preparedness for Health Emergencies, https://tinyurl.com/ gpmb-annualreport-2019 (letzter Abruf: 20.05.2020).

Harford, T. (2020): Why We Fail to Prepare for Disasters, in: Financial Times vom 16.04.2020.

Krystek, U. (2014): Risiko- und Kontinuitätsmanagement versus Resilienz- und Antifragilitäts-„,Management“: Krisenvorsorge zwischen Kontinuität und Neubeginn, in: Evertz, D./Krystek, U. (Hrsg.): Unternehmen erfolgreich restrukturieren und sanieren, Stuttgart, S. 369-403.

Krystek, U./Moldenhauer, R. (2007): Handbuch Krisen- und Restrukturierungsmanagement, Stuttgart.

Ludowig, K. (2020): Hoffnung auf Erholung im Juli, in: Handelsblatt vom 31.03.2020, S. 21.

Meissner, J./Hunziker, S. (2017): Organisationales Resilienzmanagement, in: Controlling, 29 (3), S. 14-21.

Neuhaus, C. (2006): Zukunft im Management. Orientierung für das Management von Ungewissheit in strategischen Prozessen, Heidelberg.

Reimer, M./Schäffer, U./Weber, J. (2020): Die Corona-Krise: Wie schätzen Finanzvorstände und Controller aktuell die Lage in ihren Unternehmen ein? Blitzstudie des WHU Controller Panels in Zusammenarbeit mit dem ICV, März 2020, Vallendar.

Taleb, N. N. (2007): The Black Swan. The Impact of the Highly Improbable, New York.

Taleb, N. N. (2012): Antifragile. Things That Gain from Disorder, New York. Deutsche Ausgabe: Antifragilität. Anleitung für eine Welt, die wir nicht verstehen, München 2013.

Ulrich, P./Barth, J./Lehmann, S. (2018): Stand des Risikomanagements in der Praxis, in: Krisen-, Sanierungs- und Insolvenzberatung (KSI), 18 (4), S. 154-160.

Wucker, M. (2020): No, the Coronavirus Pandemic Wasn't an 'Unforeseen Problem', in: The Washington Post vom 17.03.2020.

\section{SpringerProfessional}

Romeike, F./Hager, P. (2020): Erfolgsfaktor Risiko-Management 4.0 - Methoden, Beispiele, Checklisten. Praxishandbuch für Industrie und Handel, 4. Auflage, Wiesbaden. www.springerprofessional.de/link/18062308

Niering, C./Hillebrand, C. (2020): Wege durch die Unternehmenskrise. Sanieren statt Liquidieren - Ein Praxisleitfaden für Unternehmer und Berater, 4. Auflage, Wiesbaden. www.springerprofessional.de/link/17766624 